\title{
Induced Field Theory on the Brane World - Gravity, Extrinsic Curvature, and Gauge Fields -
}

\author{
Keiichi Akama1) and Takashi Hattori ${ }^{2)}$ \\ ${ }^{1)}$ Department of Physics, Saitama Medical College, Kawakado, Moroyama, Saitama, 350-0496, Japan \\ ${ }^{2)}$ Department of Physics, Kanagawa Dental College, Inaoka-cho, Yokosuka, Kanagawa, 238-8580, Japan \\ e-mail: ${ }^{1)}$ akama@saitama-med.ac.jp ${ }^{2)}$ hattorit@kdcnet.ac.jp
}

\begin{abstract}
We show how the gravity, extrinsic curvature, and gauge field theories are induced on dynamically localized brane world. They should obey the Gauss-Codazzi-Ricci equation in addition to their own equations of motion. As an example, we derive the solitonic solution for curved domain wall in five dimensions in terms of gravity and extrinsic curvature fields of the brane, and then derive the effective action for their field theory on the brane.
\end{abstract}

Recently the idea of the brane world attracts much attention in connection with the expectation for "large scale extra dimensions" to solve the hierarchy problems between the weak interaction scale and the Planck scale, and other problems [1,2,3, 1 . The idea that our world is an embedded object in a higher dimensional spacetime is very old. The early works were connected with embeddability problem of a Riemanian spacetime in a higher dimensional spacetime [5]. Joseph presented a picture of dynamical trapping of matters by a potential valley along the embedded world [6]. Regge and Teitelboim pointed out that we should adopt the metric but not the position of the brane as the dynamical variable to have ordinary theory of gravity [7]. Maia investigated symmetry aspects of embedded spacetimes [8]. Field theoretical trapping mechanisms of the world were considered by one of the present author [9], and Rubakov and Shaposhnikov [10], independently. The former used, as an example, the Nielsen-Olesen vortex type mechanism to trap the world on the brane, and the gravity is naturally described by the induced metric, whose kinetic term (Einstein gravity term) is induced through the quantum fluctuations. On the other hand, the latter adopted the example of the domain-wall type solution with special interest in the trapped bosons and chiral fermions on the flat brane. Gravitational trapping is considered by Visser [11. Various models were investigated in connection with various problems, such as chiral anomaly [12], supermembrane [13], and others [14,15]. It turned out that the D-brane play important rolls in the superstring theory [16], or in reducing it from the M-theory [17]. Triggered by the ideas of large extra dimension [1.2. 3 : 4 , an explosion of papers came out including phenomenological [18, cosmological [19], and theoretical investigations 20]. Besides the various possibilities as are recently studied, the idea of the brane world is basically important because it gives an alternative to the Klein-type compactification [21] to hide the Kaluza-type extra dimensions [22], which become necessary for various theoretical reasons. Other important applications of the studies of physics on the brane are those to the lower dimensional objects such as domain walls, vortices, topological defects, etc. in the condensed matter systems [23]. Here we investigate how and what field theoretical ingredients are induced on the brane world. We show that it gives a unified picture for gravity and gauge fields with additional constraints from the Gauss-Codazzi-Ricci equations.

If our world is really on a brane localized by some higher dimensional dynamics, the gravitation on the brane should be caused by dynamical deformations of the brane itself. In addition to the gravitational fields, the deformation of the brane give rise to the extrinsic curvature and the normal connection, which, respectively, behave like a tensor field and a gauge field on the brane. This suggests a natural and challenging scenario of geometrical unification of the gravitational and the gauge fields [8,15]. We call them unified because they are described by different components of the same connection from the whole spacetime viewpoints, as we will explain below.

We consider a $p+q+1$-spacetime with the coordinate system $X^{M}(M=0, \cdots, p+q)$ equipped with the local Lorentz frame specified by the vielbein $E_{A M}$. Here and hereafter Capital suffices run 0 to $p+q$, and $A, B, C, \cdots, J$ stand for local Lorentz indices, while $L, M, N, \cdots, Z$, for spacetime ones. We use the metric $G_{M N}=E_{A M} E_{N}^{A}$ and its inverse $G^{M N}$ to raise and lower spacetime indices, use $\eta_{A B}=\operatorname{diag}(1,-1, \cdots,-1)=\eta^{A B}$ to raise and lower local Lorentz indices, and use the vielbein $E_{A M}$ and its inverse $E^{A M}$ (such that $E_{A M} E^{B M}=\delta_{A}^{B}$ ) to convert spacetime indices to local Lorentz ones, and vice versa.

For a given $p$-brane, let us take a coordinate system $x^{M}$ $(M=0, \cdots, p+q)$ such that $x \underline{\mu}=0$ for $\mu=p+1, \cdots, p+q$ on the brane. Then we can use $x^{\mu}(\bar{\mu}=0,1, \cdots, p)$ as the coordinate on the brane. Hereafter the capital indices like $A, M$, etc. run over the range $0,1, \cdots, p+q$ (whole spacetime), lower case indices without underline like $\mu$, $a$, etc. run over the range $0,1, \cdots, p$ (our spacetime), and lower case indices with underline like $\underline{\mu}, \underline{a}$, etc. run over the range $p+1, \cdots, p+q$ (extra dimensions). The com- 
ponents $E_{a \mu}$ of the whole-spacetime vielbein $E_{A M}$ at the $p$-brane $\left(x^{\underline{\mu}}=0\right)$ becomes the brane vielbein, which we denote by $e_{a \mu}$, i.e. $e_{a \mu}=\left.E_{a \mu}\right|_{x \underline{\mu}=0}$. Similarly for its inverse $e^{a \mu}=\left.E^{a \mu}\right|_{x \underline{\mu}=0}$, and for the brane metric tensor $g_{\mu \nu}=e_{a \mu} e^{a}{ }_{\nu}=\left.G_{\mu \nu}\right|_{x \underline{\mu}=0}$, and $g^{\mu \nu}=\left.G^{\mu \nu}\right|_{x^{\underline{\mu}}=0}$. We use the brane metric $g_{\mu \nu}$ and its inverse $g^{\mu \nu}$ to raise and lower spacetime indices of the quantities on the brane, use $\eta_{a b}=\operatorname{diag}(1,-1, \cdots,-1)=\eta^{a b}$ to raise and lower local Lorentz indices of the quantities on the brane, and use the vielbein $e_{a \mu}$ and its inverse $e^{a \mu}$ to convert spacetime indices to local Lorentz ones of the quantities on the brane, and vice versa. We should be careful not to confuse the whole-spacetime quantities and brane quantities in the raising, lowering and converting their indices.

Let $\Omega_{A B M}$ be the connection form of the local Lorentz group $S L(p+q, 1)$, and $\mathcal{R}_{N S T}^{M}$ be the curvature tensor of the whole spacetime. Then, they are related by

$$
\mathcal{R}_{A B M N}=\Omega_{A B[M, N]}+\Omega_{C A[M} \Omega^{C}{ }_{B N]},
$$

where the square brackets [ ] in the array of the suffices indicate anti-symmetrization of the suffices, i.e. we subtract the term with the suffix at the right of [ and that at the left of ] exchanged, and the comma in the array of the suffices indicates the differentiation with respect to the spacetime coordinate components indicated by the suffices after the comma.

We denote by $\omega_{A B \mu}$ the whole space connection form $\Omega$ at the brane: $\omega_{A B \mu}=\left.\Omega_{A B \mu}\right|_{x \underline{\mu}=0}$. Then the curvature tensor $R^{\mu}{ }_{\nu \rho \sigma}$ of the $\mathrm{p}+1$ spacetime on the brane is given by

$$
R_{a b \mu \nu}=\omega_{a b[\mu, \nu]}+\omega_{c a[\mu} \omega_{b \nu]}^{c}
$$

in terms only of the tangent-tangent components of $\omega_{A B \mu}$. The components are nothing but those of the connection form of the local Lorentz group $S L(p, 1)$ on the brane world. The normal-tangent components $\omega_{\underline{a} b \mu}$ are those of (index-converted form of) the extrinsic curvature $b_{\underline{a} \mu \nu}: \omega_{\underline{a} b \nu}=b_{\underline{a} b \nu}=b_{\underline{a} \mu \nu} e_{b}^{\mu}$. The normal-normal components $\omega_{\underline{a b} \mu}$ are those of the normal connection $A_{\underline{a b} \mu}$, which play the roles of the gauge fields of the normal space rotation group $O(q)$. The curvature tensor with respect to the $O(q)$ gauge transformation is the field strength of the gauge field:

$$
F_{\underline{a b} \mu \nu}=A_{\underline{a b}[\mu, \nu]}+A_{\underline{c a}[\mu} A_{\underline{c} \underline{c}]} .
$$

Then (11) implies that

$$
\omega_{A B[\mu, \nu]}+\omega_{C A[\mu} \omega_{B \nu]}^{C}=\left.\mathcal{R}_{A B \mu \nu}\right|_{x \underline{\mu}=0},
$$

which gives constraints among the brane metric $g_{\mu \nu}$ (or the brane vielbein $e_{a \mu}$ ), the extrinsic curvature $b_{\underline{a} \mu \nu}$, and the normal-connection gauge field $A_{\underline{a b} \mu}$ in terms of the $p+q+1$ curvature at the brane. In fact, the tangent-tangent $(a b)$, tangent-normal $(a \underline{b})$, and normalnormal $(\underline{a b})$, components of (何) are rewritten as

$$
\begin{aligned}
& R_{a b \mu \nu}+b_{\underline{c} a[\mu} b_{b \nu]}^{c}=\left.\mathcal{R}_{a b \mu \nu}\right|_{x \underline{\mu}=0}, \\
& b_{\underline{a} b[\mu, \nu]}+b_{c \underline{a}[\mu} \omega_{b \nu]}^{c}+A_{\underline{c a}[\mu} b_{b \nu]}=\left.\mathcal{R}_{\underline{a} b \mu \nu}\right|_{x \underline{\mu}=0}, \\
& F_{\underline{a} \underline{\mu} \mu \nu}+b_{c \underline{a}[\mu} b_{\underline{b} \nu]}^{c}=\left.\mathcal{R}_{\underline{a b} \mu \nu}\right|_{x \underline{\mu}=0},
\end{aligned}
$$

which are called Gauss, Codazzi, and Ricci equations, respectively. On the other hand, for the whole-spacetime with constant curvature, these equations are the integrability condition for the solution for the $p$-brane to exist uniquely up to global translations and rotations for a given set of the $e_{a \mu}, b_{\underline{a} \mu \nu}$, and $A_{\underline{a b} \mu}$. Owing to this theorem, we can adopt the the $e_{a \mu}, b_{\underline{a} \mu \nu}$ and $A_{\underline{a b} \mu}$ as the dynamical variables of the $p$-brane system instead of the position $y\left(x^{\mu}\right)$ avoiding the Regge-Teitelboim problem [7].

We know various models which has solitonic solution where fields are localized in the neighborhood of some lower dimensional subspace. For example, we have the kink solution for a scalar field in a double well potential $(q=1)$, the Neilsen-Olesen vortex solution in the Higgs model with $U(1)$ gauge field $(q=2)$, the Skirmion solution with massive scalar fields $(q=3)$, etc.. In many cases, the flat space solution is well known. So here we develop a general formalism to derive the curved brane solution from the well known flat-space solution. Consider the system of the fields $\Phi$ (in general, including higher spin and spinor fields) with the flat-space action

$$
S=\int L\left(\eta_{a \mu}, \Phi, \partial_{k} \Phi\right) d_{p+q+1} X,
$$

which has some symmetry with non-symmetric potential minima. Let us assume that the equation of motion of (8) has a static soliton solution $\Phi=\Phi_{\text {sol }}\left(x^{\underline{a}}\right)$ such that

$$
\Phi_{\mathrm{sol}} \sim \Phi_{\mathrm{min}}+O\left(e^{-\delta}\right) \text { for } r=\sqrt{-x^{\underline{a}} x_{\underline{a}}} \rightarrow \infty,
$$

where $\delta$ is a constant corresponding to the soliton size, and $\Phi_{\min }$ is a singular function which takes the value with the potential minimum except at $r=0$ and is singular at $r=0$. Around the static solution $\Phi=\Phi_{\text {sol }}\left(x^{\underline{a}}\right)$, we can find small fluctuation modes $\xi$ for which $\Phi_{\text {sol }}+\xi$ is a solution of the linearized equation of motion under the assumption that $\xi$ is very small. If the action has some symmetry, there exist zero-mode solutions associated with the symmetry. They are still static implying zero additional energy to that of $\Phi_{\text {sol }}$. Among them the zero-mode of the translation invariance in the extra spase is given by $\xi_{\underline{a}}=\partial_{\underline{a}} \Phi_{\mathrm{sol}}$. Other types of the small fluctuations are non-static ones where $\xi$ depends also on $x^{\mu}$, and has additional energies.

Now we seek for the solution localized in the neighborhood of the given $p$-brane specified by the vielbein $e_{a \mu}$, the extrinsic curvature $b_{\underline{a} \mu \nu}$, and the normal connection gauge field $A_{\underline{a b} \mu}$. We assume the curvature $R_{a b \mu \nu}$, the extrinsic curvature $b_{a \mu \nu}$, and the normal connection gauge field $A_{\underline{a b \mu} \mu}$ are small in the scale of the soliton size $\delta$. This 
is an safe and plausible assumption from both cosmological and field theoretical points of view.

We assume that the whole spacetime is flat, for simplicity. Then, we specify the curvilinear coordinate system $x^{M}$ a little more precise by the transformation:

$$
\vec{X}=\vec{y}\left(x^{\mu}\right)+x^{\underline{a}} \vec{n}_{\underline{a}}\left(x^{\mu}\right),
$$

where $\vec{X}=\left(X^{0}, X^{1}, \cdots, X^{p+q}\right)$ is the cartesian coordinate, $\vec{y}\left(x^{\mu}\right)$ is the position of the brane in termes of the parameter $x^{\mu}$, and $\vec{n}_{\underline{a}}\left(x^{\mu}\right)$ are the normal vectors. In general the transformation (10) becomes singular at some large $|x \underline{a}|$. It is safe now, however, owing to the rapidly approaching property in (9) and assumption of small $R_{a b \mu \nu}, b_{\underline{a} \mu \nu}$, and $A_{\underline{a b} \mu}$. Then the vielbein $E_{A M}=\vec{n}_{A} \vec{X}_{, M}$ is given by

$$
\left(\begin{array}{ll}
E_{a \mu} & E_{a \underline{\mu}} \\
E_{\underline{a} \mu} & E_{\underline{a \mu}}
\end{array}\right)=\left(\begin{array}{cc}
e_{a \mu}-x_{\underline{b}} b_{\underline{b} a \mu} & 0 \\
-x \underline{b} A_{\underline{b a} \mu} & \eta_{a \mu}
\end{array}\right) .
$$

The action becomes

$$
S=\int E L\left(E_{A M}, \Phi, D_{M} \Phi\right) d_{p+q+1} x
$$

in the curvilinear coordinate specified by the vielbein $E_{A M}$, where $E=\operatorname{det} E_{A M}$, and $D_{M}$ is the covariant differentiation operator written in terms of the connection $\omega_{A B \mu}$ according to the spins of the ingredients of the field $\Phi$.

If the $p$-brane is curved, the solution $\Phi_{\text {sol }}$ is no longer a solution of the equation of motion. The small correction is expected to have an asymptotic form proportional to the translation zero-mode. Then we parametrize the small distortion of $\Phi_{\text {sol }}$ as

$$
\Phi_{\text {brane }}\left(x^{M}\right)=\Phi_{\text {sol }}\left(x^{\underline{\mu}}\right)+\chi \underline{\mu} \underline{\partial}_{\underline{\mu}} \Phi_{\text {sol }}\left(x^{\underline{\mu}}\right),
$$

where $\chi^{\underline{\mu}}$ is the small coefficient (in general matrix because $\Phi$ is a system of fields) which depends on both $x^{\mu}$ and $x \underline{\mu}$. Because the solution rapidly approaches its asymptotic value for $x \underline{\mu}>>\delta$, only the region with small $x \underline{\underline{\mu}}$ is important. So we expand $\chi$ in terms of $x \underline{\mu}$ :

$$
\chi^{\underline{\mu}}=\chi^{(0) \underline{\mu}}+x^{\underline{\nu}} \chi_{\underline{\nu}}^{(1) \underline{\mu}}+x^{\underline{\nu}} x^{\underline{\lambda}} \chi_{\underline{\nu \lambda}}^{(2) \underline{\mu}}+\cdots,
$$

where $\chi^{(i)}(i=0,1,2, \cdots)$ are functions of $x^{\mu}$ only. The function $\chi^{(0)}$ corresponds to the shift of the position of the brane, and it should be described by other values of $e_{a \mu}, b_{\underline{a} \mu \nu}$, and $A_{\underline{a b} \mu}$. Therefore we neglect $\chi^{(0)}$ in $(14)$. Now we can expand the equation of motion in power series of $x \underline{\mu}$. The equation should hold order by order in $x-\underline{\mu}$. This gives recursive equations for the functions $\chi^{(i)}$ in terms of the vielbein $e_{a \mu}$, the extrinsic curvature $b_{a \mu \nu}$, and the normal connection gauge field $A_{\underline{a b} \mu}$. Thus we can obtain the $p$-brane solution $\Phi_{\text {brane }}\left(x^{\mu}\right)$ in the form of (13), where $\chi$ is power series of $x \underline{\mu}$ with coefficients written in terms of the parameters $e_{a \mu}, b_{\underline{a} \mu \nu}$, and $A_{\underline{a b} \mu}$ which specify the $p$-brane. If we substitute this $\Phi_{\text {brane }}\left(x^{M}\right)$ back into the action (12) and perform $x$-integration, we obtain the effective action for the quantities $e_{a \mu}, b_{a \mu \nu}$, and $A_{\underline{a b} \mu}$ on the brane world.

As $\overline{\text { an }}$ example, here we apply this procedure to the model for the scalar field $\Phi$ with a double well potential in the five dimensional spacetime [10].

$$
\begin{aligned}
& S=\int E\left[\frac{1}{2} G^{M N} \partial_{M} \Phi \partial_{N} \Phi-U(\Phi)\right] d^{5} x \\
& \text { with } U(\Phi)=\frac{1}{4} \lambda\left(\Phi^{2}-\frac{m^{2}}{\lambda}\right)^{2},
\end{aligned}
$$

where $\lambda$ and $m$ are constants. The equation of motion for $\Phi$ is given by

$$
\partial_{M} E G^{M N} \partial_{N} \Phi=-E U^{\prime}(\Phi) .
$$

We solve this for $\Phi$ with a given configuration of $E_{K M}$. In the flat case, this is known to have the domain wall solution

$$
\Phi=\Phi_{\mathrm{sol}} \equiv \frac{m}{\sqrt{\lambda}} \tanh \frac{m y}{\sqrt{2}},
$$

where we have denoted the extra dimension coordinate $x^{\underline{4}}$ by $y$. To find the solution of (17) for general $E_{K M}$, we transform the variable $\Phi$ into $\chi$ by

$$
\Phi\left(x^{\mu}, y\right)=\Phi_{\mathrm{sol}}(y)+\chi\left(x^{\mu}, y\right) \Phi_{\mathrm{sol}}^{\prime}(y)
$$

without loss of generality. Then we expand (17) using

$$
\begin{aligned}
& \chi\left(x^{\mu}, y\right)=\chi_{0}\left(x^{\mu}\right)+\chi_{1}\left(x^{\mu}\right) y+\chi_{2}\left(x^{\mu}\right) y^{2}+\cdots, \\
& \Phi_{\mathrm{sol}}=m^{2}\left(y-m^{2} y^{2} / 6+\cdots\right) / \sqrt{2} \lambda, \\
& E=e\left[1-b_{\mu}^{\mu}-\left\{b_{\mu}^{\nu} b_{\nu}^{\mu}-\left(b_{\mu}^{\mu}\right)^{2}\right\} y^{2} / 2+\cdots\right], \\
& G^{\mu \nu}=g^{\mu \nu}+2 b^{\mu \nu} y+3 b_{\lambda}^{\mu} b^{\lambda \nu} y^{2}+\cdots,
\end{aligned}
$$

where $b_{\mu \nu}$ is the extrinsic curvature (the extra-dimension index $\underline{4}$ of $b_{\underline{4}} \mu \nu$ is suppressed). The normal connection gauge field $\hat{A}_{\underline{a b} \mu}$ does not appear in this particular example, because the number of the extra dimensions is one and no rotation ocuurs. The equation of motion (17) gives the recursion formulae for $\chi_{i}$ 's:

$$
\begin{aligned}
& -b_{\mu}^{\mu}\left(1+\chi_{1}\right)+2 \chi_{2}-m^{4} \chi_{0}^{3} / 2=e^{-1} \partial_{\mu} e g^{\mu \nu} \partial_{\nu} \chi_{0} \\
& -b\left(2 \chi_{2}-m^{2} \chi_{0}\right)-\left\{b_{\mu}^{\nu} b_{\nu}^{\mu}-\left(b_{\mu}^{\mu}\right)^{2}\right\}\left(1+\chi_{1}\right)+6 \chi_{3} \\
& -2 m^{2} \chi_{1}-3 m^{4} \chi_{0}^{2}\left(1+\chi_{1}\right)-b\left(2 \chi_{2}-m^{4} \chi_{0}^{3}\right) \\
& =e^{-1} \partial_{\mu} e\left[g^{\mu \nu} \partial_{\nu} \chi_{1}+\left(2 b^{\mu \nu}-b g^{\mu \nu}\right) \partial_{\nu} \chi_{0}\right] \\
& \ldots \quad \ldots \quad \ldots \quad \ldots
\end{aligned}
$$

which are solved to give

$$
\begin{aligned}
& \chi_{2}=b_{\mu}^{\mu}\left(1+\chi_{1}\right) / 2+m^{4} \chi_{0}^{3} / 4+e^{-1} \partial_{\mu} e g^{\mu \nu} \partial_{\nu} \chi_{0} / 2, \\
& \chi_{3}=\left[b_{\mu}^{\nu} b_{\nu}^{\mu}+\left(b_{\mu}^{\mu}\right)^{2}-e^{-1} \partial_{\mu} e\left(2 b^{\mu \nu}-b g^{\mu \nu}\right) \partial_{\nu} \chi_{0}\right] / 6 \\
& +m^{4} \chi_{0}^{2} / 4+b\left(-m^{2} \chi_{0}+m^{4} \chi_{0}^{3} / 2\right) / 6+m^{4} \chi_{0}^{2} \chi_{1} / 4
\end{aligned}
$$




$$
+\left[e^{-1} \partial_{\mu} e g^{\mu \nu} \partial_{\nu} \chi_{1}+2 m^{2} \chi_{1}+\left\{b_{\mu}^{\nu} b_{\nu}^{\mu}+\left(b_{\mu}^{\mu}\right)^{2}\right\} \chi_{1}\right] / 6,
$$

The solution involves arbitrary functions $\chi_{0}\left(x^{\mu}\right)$ and $\chi_{1}\left(x^{\mu}\right)$. Among them $\chi_{0}\left(x^{\mu}\right)$ shifts the position of the brane, and the effects should be considered with another configuration of $e_{k \mu}$ and $b_{\mu \nu}$. So we omit it. In other words, it is kept vanishing by the center-of-mass constraint of the brane slices along the extra dimension. On the other hand $\chi_{1}$ changes the length scale of the extra dimension at the brane. This is arbitrary and we can choose the scale unity all over the brane, so that $\chi_{1}$ vanishes everywhere. Thus we finally obtain the curved brane world solution

$$
\begin{aligned}
& \Phi=\Phi_{\text {brane }} \equiv \\
& \Phi_{\text {sol }}+\left[\frac{1}{2} y^{2} b_{\mu}^{\mu}+\frac{1}{6} y^{3}\left\{\left(b_{\mu}^{\mu}\right)^{2}+b_{\mu \nu} b^{\mu \nu}\right\}+\cdots\right] \Phi_{\text {sol }}^{\prime}
\end{aligned}
$$

We substitute this $\Phi_{\text {brane }}$ back into the action (15). After some manipulation, we obtain

$$
\begin{gathered}
S=\frac{\lambda}{4} \int E\left(\Phi_{\text {brane } \left.^{4}-\frac{m^{4}}{\lambda^{2}}\right) d^{5} x}\right. \\
=\frac{\lambda}{4} \int e\left[\left(1-\frac{2 b_{\mu}^{\nu} b_{\nu}^{\mu}-3\left(b_{\mu}^{\mu}\right)^{2}}{2}\right)\left(\Phi_{\text {sol }}-\frac{m^{4}}{\lambda^{2}}\right)\right. \\
\left.+\frac{3}{2}\left(b_{\mu}^{\mu}\right)^{2} \Phi_{\mathrm{sol}}^{2} \Phi_{\text {sol }}^{\prime}{ }^{2}\right] d y d^{4} x .
\end{gathered}
$$

The integrand of $(29)$ is localized around the brane. This means that the fields $e_{a \mu}$ and $b_{\mu \nu}$ are trapped by the brane. So we perform the $y$-integrations

$$
\begin{aligned}
& \int\left(\Phi_{\mathrm{sol}}-\frac{m^{4}}{\lambda^{2}}\right) d y=-\frac{8 \sqrt{2} m^{3}}{3 \lambda^{2}} \\
& \int y^{2}\left(\Phi_{\mathrm{sol}}-\frac{m^{4}}{\lambda^{2}}\right) d y=-\frac{4 \sqrt{2} m}{3 \lambda^{2}}\left(1+\frac{\pi^{2}}{3}\right) \\
& \int y^{4} \Phi_{\mathrm{sol}}^{2} \Phi_{\mathrm{sol}}^{\prime} d y=-\frac{2 \sqrt{2} m}{\lambda^{2}}\left(-\frac{2}{5}+\frac{7 \pi^{4}}{900}\right)
\end{aligned}
$$

and we finally obtain the effective action for the quantities $e_{a \mu}$ and $b_{\mu \nu}$ on the brane world:

$$
\begin{aligned}
S=\int e[- & \frac{2 \sqrt{2} m^{3}}{3 \lambda}+\frac{\sqrt{2} m}{3 \lambda}\left(1+\frac{\pi^{2}}{3}\right) b_{\mu}^{\nu} b_{\nu}^{\mu} \\
& \left.+\frac{\sqrt{2} m}{\lambda}\left(-\frac{4}{5}+\frac{\pi^{2}}{6}+\frac{7 \pi^{4}}{1200}\right)\left(b_{\mu}^{\mu}\right)^{2}\right] d^{4} x .
\end{aligned}
$$

They are the cosmological term and the mass terms of the field $b_{\mu \nu}$.

Now we return to the general formalism. Let $\xi_{n}(n=$ $1,2, \cdots)$ be the small fluctuation modes of the flat space solution. We expand the small fluctuation of the field $\Phi$ in terms of $\xi_{n}$ as

$$
\Phi=\Phi_{\text {brane }}+\sum_{n} \varphi_{n}\left(x^{\mu}\right) \xi_{n}\left(x^{\underline{\mu}}\right) .
$$

Here $\varphi_{n}$ and $\xi_{n}$ are, in general, with further implicit indices which denote various spin and other internal quantum numbers. It can involve fermionic modes also. We substitute this into the action (12). Among them, the terms concerning the low-lying states are localizeded around the brane by the solitonic potential. We perform the $x \underline{\mu}$-integration for the localized sector. Then we obtain the effective action $S_{\text {eff }}$ for $e_{a \mu}, b_{\underline{a} \mu \nu}, A_{\underline{a b} \mu}$, and the localized modes on the $p$-brane.

So far we have treated the fields $E_{A M}$ in the $p+q+1$ spacetime and $e_{a \mu}, b_{\underline{a} \mu \nu}$, and $A_{\underline{a b} \mu}$ on the brane as the given external fields. To incorporate their quantum dynamics, we should path-integrate the generating functional over all the possible configurations. We can add the kinetic term of $E_{A M}$ in the $p+q+1$-spacetime because it is taken as an elementary field. Then we will have some equation which specifies the configuration of $E_{A M}$ in the $p+q+1$-spacetime. If the quanta (gravitons etc.) of the system is massless, the coupling of the interaction should not be so strong as to violate the observed level of the effective energy conservation on the brane. On the other hand, we should path-integrate the generating functional with respect to $e_{a \mu}, b_{\underline{a} \mu \nu}$, and $A_{\underline{a b} \mu}$ on the brane to incorporate all the possible and independent configurations of the brane.

The effective action derived as above has no kinetic term of $e_{a \mu}, b_{\underline{a} \mu \nu}$, and $A_{\underline{a b} \mu}$. The kinetic terms are induced through the quantum fluctuations of the trapped matter field $\varphi$ on the brane, as has been extensively studied in the induced field theories 24,25] and the induced gravity theories [26,27,28,29]. In general the quantum fluctuations have the form like

$$
\begin{aligned}
& e\left[c_{0}+c_{1} R+\cdots+c_{2}\left(b_{\underline{a} \mu \nu}\right)^{2}+c_{3}\left(\partial_{\rho} b_{\underline{a} \mu \nu}\right)^{2}+\cdots\right. \\
& \left.\quad+c_{4}\left(F_{\underline{a} \boldsymbol{b} \mu \nu}\right)^{2}+\cdots\right],
\end{aligned}
$$

where $c_{i}$ are calculable constants. The field theoretical calculations are found in the literature. The values of $c_{0}$, $c_{1}, c_{2}$, and $c_{3}$,respectively, contain quartic, quadratic, logarithmic, and logarithmic divergences in the ultraviolet region, which we cutoff at around the scale of asymptotic field value $\left|\Phi_{\min }\right|$. Such a cutoff is naturally expected in the present model, since very high excitation above the threshold of $\left|\Phi_{\min }\right|$ spread into the extra dimensions and blind to the position of the brane. Then the effective action on the brane gives the equations of motion of $e_{a \mu}$, $b_{\underline{a} \mu \nu}$, and $A_{\underline{a b} \mu}$ on the brane. The cosmological constant should be fine-tuned.

In conclusion, on the brane world localized by the solitonic solution,

(i) the gravitational and the gauge fields are induced through the deformation of the brane,

(ii) besides them the extrinsic curvature field are induced through the deformation of the brane,

(iii) the gravitational, the gauge and the extrinsic curvature fields should obey the Gauss-Codazzi-Ricci equations in addition to the ordinary equations of motion, 
(iv) the kinetic terms of the gravitational, the gauge and the extrinsic curvature fields are induced through the quantum fluctuations of the trapped matter fields.

One of the authors (K.A.) would like to thank Dr. H. Mukaida for discussions. hep-ph/0006344.

[19] For example, T. Nihei, Phys. Lett. B465, 81 (1999). R. Maartens, D. Wands, B. A. Bassett, and I. Heard, hep-ph/9912464: K. Koyama and J. Soda, Phys. Lett. B483, (2000) 432; U. Guenther and A. Zhuk, Phys.Rev. D61, (2000) 124001; H. Ishihara, gr-qc/0007070: P. Bowcock, C. Charmousis, and R. Gregory, hep-th/0007177; P. Binetruy and J. Silk, astro-ph/0007452; A. Mazumdar, hep-ph/0008087; L. Anchordoqui and K. Olsen, hepth/0008102.

[20] For example, J. Kubo, H. Terao, and G. Zoupanos, Nucl. Phys. B574, 495 (2000) J. Hisano and N. Okada, Phys.Rev. D61 (2000) 106003; I. Oda, Phys. Lett. B472, 59(2000); B480, 305 (2000); hep-th/0006203; hepth/0008012: hep-th/0008055; M. Ooho and K. Shiraishi, gr-qc/9912110; M.J. Duff, hep-th/9912164; D. Youm, Nucl. Phys. B576, 123 (2000); hep-th/0001166 Z. Chacko and A. E. Nelson, hep-th/9912186; E. Dudas, hepph/0006190: T. Gherghetta, E. Roessl, and M. Shaposhnikov, hep-th/0006251; I. Giannakis and H.-c. Ren, hep-th/0007053; R. Dick, hep-th/0007063; P. Dimopoulos, K. Farakos, A. Kehagias, and G. Koutsoumbas, hep-th/0007079, S. L. Dubovsky, V. A. Rubakov, and P. G. Tinyakov, hep-ph/0007179; S. Nojiri and S. D. Odintsov, hep-th/0007205: K. G. Selivanov, hepth/0007246; C. Barcelo and M. Visser, gr-qc/0008008; G. Dvali and G. Gabadadze, hep-th/0008054; N. E. Mavromatos and J. Rizos, hep-th/0008074; S. Randjbar-Daemi and M. Shaposhnikov, hep-th/0008079; hep-th/0008087.

[21] O. Klein, Zeitschrift für Physik 37895 (1926).

[22] Th.Kaluza, Sitzungsberichte der K.Preussischen Akademite der Wissenschaften zu Berlin p.966 (1921).

[23] H. Arodz, Nucl. Phys. B509, 273-293 (1998); Acta Phys. Polon. B29, 3725-3737 (1998).

[24] Y. Nambu and G. Jona-Lasinio, Phys. Rev. 122 (1961) 345; J. D. Bjorken, Ann. Phys. 24 (1963) 174; H. Terazawa, Y. Chikashige and K. Akama, Phys. Rev. D15 (1977) 480.

[25] For example, K. Akama, Phys. Rev. Lett. 76 (1996) 184; K. Akama and T. Hattori, Phys. Lett. B392 (1997) 383;B445 (1998) 106; T. Hattori, Kanagawa Dental College preprint (2000); references therein.

[26] A. D. Sakharov, Dokl. Akad. Nauk SSSR 177, 70 (1967) [Sov. Phys. Dokl. 12, 1040 (1968)].

[27] K. Akama, Y. Chikashige, T. Matsuki and H. Terazawa, Prog. Theor. Phys. 60, 868 (1978).

[28] K. Akama, Prog. Theor. Phys. 60, 1900 (1978).

[29] A. Zee, Phys. Rev. Lett. 42, 417 (1979); S. L. Adler, Phys. Rev. Lett. 44, 1567 (1980). 